\title{
SCREENING ALEXANDRIA PORT SIMULATION MODEL BY USING SEQUENTIAL BIFURCATION PROCEDURE
}

\author{
Ragheb, M. ; Rekab, K.** \&adj, L.** \\ *College of Management and Technology, \\ Arab Academy for Science, Technology and Maritime Transport, Egypt \\ ${ }^{* *}$ Department of Mathematics and Statistics, University of Missouri-Kansas State, USA \\ ${ }^{* * *}$ School of Business Administration, American University in Dubai, UAE \\ E-Mail: ltadj@aud.edu
}

\begin{abstract}
Screening simulation experiments is intended to eliminate unimportant factors from a simulation model. It usually results in a short list of important factors, so that effort may be concentrated upon this list. Sequential bifurcation (SB) procedure, developed by Bettonvil and Kleijnen [1], is a screening method that has proved to be efficient and effective. In this study, the SB procedure is used to determine the most important factors of the port of Alexandria, Egypt simulation model. Sixteen out of 44 factors are considered important. In addition, Cioppa [2] procedure is used to verify that the assumed unimportant factors by the SB procedure are really unimportant. This procedure is used to confirm that the suspected non-influential (unimportant) factors do not significantly affect the response output of interest. This procedure emphasized that the set of unimportant factors determined by the SB procedures is really unimportant.

(Received in November 2009, accepted in March 2010. This paper was with the authors 1 month for 1 revision.)
\end{abstract}

Key Words: Simulation, Screening, Sequential Bifurcation

\section{INTRODUCTION}

Screening in simulation is to find the most important input parameters. It is the first phase of a design of experiments study for simulation models. Screening is the process of searching for the few important factors among the great many potentially important factors that affect a system's performance. Therefore, the purpose of screening is to eliminate negligible factors so that efforts may be concentrated upon just the important input parameters. The idea of screening is based on the "parsimony principle" which says that only few factors are responsible for most of the effect in response variable [2].

Trocine and Malone [3] determined four criteria for selecting a screening method. These criteria are efficiency, effectiveness, robustness and ease of use. The efficiency of the screening method is measured by the number of required runs. The efficient screening method is the one that needs a manageable number of runs. Effectiveness of the screening method is the ability of determining the important factors of the simulation experiment. The robustness of the screening method is how the method works without prior knowledge of the problem. A robust method is the one that works well without conditions. The last criterion is the ease of use; it is desirable but not necessary. It can be dropped for the sake of efficiency, effectiveness and robustness.

According to Trocine and Malone [4], the choice of a screening method depends on the number of factors in the model. For few factors (less than or equal to five), the full factorial $\left(2^{k}\right)$ design is the best. The fractional factorial $\left(2^{k-p}\right)$ is the best for fewer than 20 factors. For 
thousands of factors, the Iterated fractional factorial design (IFFD) is the most appropriate. For dozens to hundreds, the 2-stage group screening and sequential bifurcation works well.

There are many screening methods. Some procedures are specifically intended to facilitate large-scale experiments on simulation models by taking advantage of the sequential nature of simulation experiments. Kleijnen et al. [5] provide a general description of the design and analysis of simulation experiments.

Recently, group screening has been developed for simulation experiments. One such procedure is sequential bifurcation (SB). Originally, the sequential bifurcation technique for deterministic simulation models was developed by Bettonvil [6] in his doctoral dissertation which was summarized by Bettonvil and Kleijnen [1] and by Kleijnen et al. [7]. They assume important factors are sparse, that the direction of all factor effects is known, and that a main effects metamodel is a reasonable approximation of the simulation model over the region of an exploration. Cheng [8], who assumed that the errors are normally distributed with constant variance, extended SB to stochastic simulation models.

Kleijnen et al. [5] proposed the use of SB for screening stochastic simulation models. This technique was proved efficient and effective; that is, it can find all the important factors in a few numbers of replications. Deterministic SB performs well when the factor effects are initially ordered according to increasing or decreasing values of the unknown factor effects. On the other hand, there is no guarantee of the performance for the stochastic simulation experiments.

Wan et al. [9] propose another version of SB called controlled sequential bifurcation (CSB) procedure. The purpose of the CSB is to overcome the shortcoming of the SB procedure. In CSB, the analyst specifies two thresholds. The lower threshold indicates the level that the effect must reach to be considered important. The effects larger than the higher threshold are considered critical. CSB is based on the same assumptions of the SB procedure.

Sanchez et al. [10] proposed a two-phase screening procedure for simulation experiments. In phase 1, an efficient fractional factorial is conducted to classify the factors into groups according to the signs and magnitudes of their estimated effects. This classification is the basis for applying sequential CSB in the second phase of the simulation experiment.

In this study, the sequential bifurcation method, developed by Bettonvil and Kleijnen, is used for screening the port simulation model developed by Ragheb [11]. The rest of the paper is organized as follows. In Section 2 we review the key literature on simulation model validation techniques and in Section 3 we describe the SB technique. In Section 4, the SB procedure is used to determine the most important factors of the port of Alexandria, Egypt simulation model. Section 5 concludes the paper.

\section{LITERATURE REVIEW}

Nelson [12] described the basic principles for designing statistically efficient simulation experiments and controlling the experiment error. This research concentrated on the simulation experiments performed to compare the performance of two or more systems, whether for terminating or steady-state simulation. Simulation experiments that estimate performance parameters defined with respect to pre-specified initial and final conditions are called terminating simulation experiments. Simulation experiments that estimate performance parameters defined over an infinite time horizon are called steady-state simulation experiments. Designing simulation experiments that control the error requires obtaining some estimate of the error. This means that a simulation experiment must be performed in (at least) two stages; an exploratory experiment, and a designed experiment. The purpose of the exploratory experiment is to provide the information for planning the design experiment, which in turn provides the required comparison. 
Donohue [13] discussed the tactical and strategic issues related to experimental designs for simulation models. According to this research, the statistical approach to the design of experiments for simulation models includes the following steps: (1) state the problem requiring experimentation and state the objective of the study (e.g., prediction, optimization, sensitivity analysis); (2) choose the factors (controllable input variables); (3) determine the response variable (simulation output variable); (4) determine the domain of each factor within which the simulation will run; (5) specify the region of interest (a subregion of the region determined in (4)); (6) choose the statistical model to be used (e.g., ANOVA, regression analysis); (7) select criteria for choosing an experimental design (e.g., minimize generalized variance, minimize mean squared error); (8) select a suitable experimental design class (e.g., factorial, Latin square, central composite); (9) determine the level of factors for each experimental run; (10) perform the experiments and collect data; (11) analyze and summarize the data, draw inferences and conclusions.

Kleijnen [14-15] recommended the following sequence for analysis and evaluation of simulation models: (1) validation and verification; (2) screening; (3) sensitivity or what-ifanalysis; (4) uncertainty or risk analysis; (5) optimization. The validation and verification of the simulation model can be done by using the design of experiments if there are no data on the input/output, or using special types of regression analysis if there is enough data. The screening is the search for few (say) $k$ important factors among the many (say) $N$ potentially important factors. This search can be done using the sequential bifurcation method. Once the important factors are identified, further analysis is recommended. Sensitivity analysis can be done by using regression analysis and design of experiments. The regression metamodel shows what factors are important. For the important environmental inputs, the analysts should try to collect data on the values that occur in practice. If they fail, then they may use uncertainty analysis. The goal of uncertainty analysis is to quantify the probability of specific output values, where the sensitivity does not tell how likely a specific result is. Optimization of simulation models can be done using RSM (response surface methodology).

Kelton [16] introduced some ideas and issues in deciding how to experiment with a simulation model. In this research, Kelton answered some of the questions that the simulation analyst deals with, such as: What model configuration should one use? How long should the runs be? How many runs should one make? How should one interpret and analyze the output? What is the most efficient way to make the runs?

Schmeiser [17] presented the simulation experiment in a good way as follows: $G \rightarrow U \rightarrow X \rightarrow Y \rightarrow \hat{\theta}$. Each of the four arrows is a deterministic function, with the randomness generator $G$ used to generate a set of random numbers $U$, which is used to generate a set of input data $X$, which is used to compute a set of output data $Y$, which is used to estimate the point estimate $\hat{\theta}$. The model is composed of two parts. The input model, corresponding to the arrow between $U$ and $X$, is the given probability model for the random variable $X$. The logic model, corresponding to the arrow between $X$ and $Y$, is the relationship between input data and output data. Schmeiser defined in this research the sources of error in simulation experiment as: (1) coding error; (2) numerical error; (3) random number error; (4) random variate error; and (5) sampling error.

Barton [18-19] defined the design of simulation experiments as a repeating cyclic process, which can be broken down into several steps: (1) state the hypothesis to be evaluated; (2) plan an experiment to test the hypothesis; (3) conduct the experiment; and (4) analyze the data from the experiment. Step 2, which is called sometimes design of simulation experiments, can also be broken into several steps: (1) define the goal of the experiment; (2) identify and classify independent and dependent variables; (3) choose a probability model for the behaviour of the simulation model; (4) choose an experimental design; and (5) validate the properties of the chosen design. 
Kleijnen et al. [7] presented a toolkit for design of simulation experiments. In this user guide, they addressed the difference between real world design of experiments and simulation design of experiments. They discussed the issues analysts should be aware of as they prepare to code, collect, and analyze output from a simulation experiment. In addition, they presented a guide for selecting the appropriate design. For more details on the design of simulation experiments techniques discussed in this study; see Kleijnen et al. [7].

\section{SEQUENTIAL BIFURCATION DESCRIPTION}

The sequential bifurcation procedure begins with all the factors as one group and tests whether or not this group of factors has an important effect. If it does, this group is split into two subgroups, which in turn are tested for importance. The procedure will continue by discarding unimportant groups and splitting important groups into smaller ones. Eventually, a list of important factors is generated [7]. Sequential bifurcation is based on two assumptions:

Assumption 1: A first order metamodel that can be augmented by a two-factor interaction is adequate to represent the relation between the input parameters (factors) and the output response (the performance indicator); that is:

$$
Y=\beta_{0}+\sum_{j=1}^{n} \beta_{j} x_{j}+\sum_{i=1}^{n} \sum_{j>i}^{n} \beta_{i, j} x_{i} x_{j}+\varepsilon
$$

where $Y$ is the output response, $x_{\mathrm{j}}$ is the value of factor $j$ standardized to range between 0 and $1, \beta_{j}$ is the main effect of factor $j, \beta_{i, j}$ is the interaction effect between factors $i$ and $j(1 \leq i<j$ $\leq n$ ), and $\varepsilon$ is the error (noise) variable rising from the use of random numbers and approximation error. Note that this model assumes the independence of the responses $Y$.

Assumption 2: the sign of the main effects are known and are nonnegative; that is:

$$
\beta_{j} \geq 0, j=1,2, \ldots, n
$$

This assumption is easy to be satisfied by defining the higher and lower limit of each factor such that changing the factor from the lower to the higher level does not decrease the expected value of the output response. This means that the lower level of each factor will result in a low expected response and the high level will result in a high-expected response [7]. Now we will define the notation of the sequential bifurcation used by the same research as follows:

- $Y(j) ; r$, where $r=1,2, \ldots, m$, is the simulated output response in replicate $r$, when factors 1 to $j$ are set at their high level denoted by +1 and the remaining factors are set at their low levels denoted by -1 .

- $\quad \beta_{i-j}$ is the sum of the main effect of factors $i$ to $j$; that is:

$$
\beta_{i-j}=\sum_{k=i}^{j} \beta_{k}
$$

An estimate of this aggregated main effect $\beta_{i-j}$, using only the output of replicate $r$, is:

$$
\hat{\beta}_{i-j ; r}=\frac{Y(j) ; r-Y(i-1) ; r}{2}
$$

And the individual main effect of factor $j$ from replicate $r$, is:

$$
\hat{\beta}_{j ; r}=\frac{Y(j) ; r-Y(j-1) ; r}{2}
$$

Note that these tow equations are valid under the no interaction assumption. The average aggregate and average individual main effects are calculated as follows: 


$$
\begin{gathered}
\overline{\hat{\beta}}_{i-j}=\sum_{r=1}^{m} \hat{\beta}_{i-j ; r} \\
\hat{\hat{\beta}}_{j}=\sum_{r=1}^{m} \hat{\beta}_{j ; r}
\end{gathered}
$$

\section{SCREENING THE PORT SIMULATION MODEL}

In this section, the sequential bifurcation method is used to determine the most important factors of a seaport simulation model. The seaport simulation model was developed by Ragheb [11] doctoral dissertation and was intended to calculate the performance indicators of the port. The designed model was proved valid for the port of Alexandria, Egypt.

Table I shows the base, low, and high values of all the controllable factors that affect the turnaround time (one of the most important performance indicators of a seaport) as an output response. The high levels $(+1)$ are the values that result in higher expected turnaround time. The low levels (-1) are the values that result in lower expected turnaround time. The high and low level values of the factors are determined as $\pm 10 \%$ of the base values for the tackle load and crane cycle time of the different cargo types. They are determined as \pm 1 of the base value for the different categories of the inner and outer pilots. For the other factors, they are determined by studying the range of the values that affect the turnaround time. For example, the low level of the number of general cargo berths is set as 20 berths. However, the high level is set as the base value 26 because any value greater than the base value does not change the turnaround time. Also decreasing the number of berths to 16 will not affect the turnaround time.

\section{Steps of the sequential bifurcation method:}

Firstly, we will assume no interaction effects between factors. Later this assumption will be dropped.

Initial step (step 0): the sequential bifurcation procedure starts with simulating the two most extreme scenarios. In scenario 1, all the factors are set at their low level; therefore, we expect the smallest expected turnaround time; for $r$ equals 10 replications we obtained $\bar{Y}(0)=$ $5976.661 \mathrm{~min}$. In scenario 2, all the factors are set at their high level; therefore, we expect the largest expected turnaround time; $\bar{Y}(44)=9327.57 \mathrm{~min}$. Therefore, the aggregate main effect of all the 44 factors is:

$$
\overline{\beta_{1-44}}=\frac{\bar{Y}(44)-\bar{Y}(0)}{2}=1675.455 \mathrm{~min}
$$

Table II shows the values of $Y(44) ; r$ and $Y(0) ; r$ of the 10 replicates as well as the values of $\hat{\beta}_{1-44} ; r$; for $r=1,2, \ldots, 10$.

To test the importance of the main effect statistically, we assume that the outputs of the 10 replicates are approximately normally and independently distributed and then we apply one tailed $t$-test (since we assume that all the signs of the main effects are positive). At the significance level $\alpha=0.1$ and 9 degrees of freedom, the critical value from the $t$ distribution is 1.383. Since the $t$-statistic (average/standard error) for the aggregate main effect is 4.57 and is greater than the critical value, we conclude that the aggregate main effect is significantly different from zero. In practice, the significance is not important for the sequential bifurcation method since we are looking for a shortlist of important factors; see [7]. 
Table I: The base value, high level and low level of controllable variables.

\begin{tabular}{|c|c|c|c|c|}
\hline No. & Variable & Base value & High level (+1) & Low level (-1) \\
\hline 1 & Speed of ships outside the port & $(8,12)$ & $(4,8)$ & $(12,16)$ \\
\hline 2 & Outer anchorage setup time & $(10,30)$ & $(30,50)$ & $(1,10)$ \\
\hline 3 & Senior outer pilot & 2 & 1 & 3 \\
\hline 4 & First outer pilot & 3 & 2 & 4 \\
\hline 5 & Second outer pilot & 1 & 0 & 2 \\
\hline 6 & Third outer pilot & 2 & 1 & 3 \\
\hline 7 & Outer launches & 6 & 5 & 7 \\
\hline 8 & Speed of ships inside the port & $(3,5)$ & $(1,3)$ & $(5,7)$ \\
\hline 9 & Inner anchorage setup time & $(10,30)$ & $(30,50)$ & $(1,10)$ \\
\hline 10 & Lashing launches & 17 & 15 & 19 \\
\hline 11 & Lashing time & $(5,10)$ & $(10,15)$ & $(1,5)$ \\
\hline 12 & Senior inner pilot & 2 & 1 & 3 \\
\hline 13 & First inner pilot & 3 & 2 & 4 \\
\hline 14 & Second inner pilot & 2 & 1 & 3 \\
\hline 15 & Third inner pilot & 3 & 2 & 4 \\
\hline 16 & Inner launches & 5 & 4 & 6 \\
\hline 17 & Tugboats & 24 & 14 & 24 \\
\hline 18 & General cargo berths & 26 & 20 & 26 \\
\hline 19 & Other cargo berths & 60 & 40 & 60 \\
\hline 20 & Time before cargo handling operation & 438 & 482 & 394 \\
\hline 21 & Bags tackle load & 1.7 & 1.53 & 1.87 \\
\hline 22 & Cartons tackle load & 1 & 0.9 & 1.1 \\
\hline 23 & Barrels tackle load & 0.8 & 0.72 & 0.88 \\
\hline 24 & Paper rolls tackle load & .73 & 0.657 & 0.803 \\
\hline 25 & Iron bars tackle load & 4.5 & 4.05 & 4.95 \\
\hline 26 & Iron rolls tackle load & 2 & 1.8 & 2.2 \\
\hline 27 & Iron pipes tackle load & 1 & 0.9 & 1.1 \\
\hline 28 & Boxes tackle load & 1 & 0.9 & 1.1 \\
\hline 29 & Bags crane cycle time & 0.3295235 & 0.362475916 & 0.2965712 \\
\hline 30 & Bales crane cycle time & 3.0308873 & 3.33397603 & 2.72779857 \\
\hline 31 & Barrels crane cycle time & 5.632503 & 6.1957533 & 5.0692527 \\
\hline 32 & Paper rolls crane cycle time & 0.3765047 & 0.41415517 & 0.33885423 \\
\hline 33 & Iron rolls crane cycle time & 3.7530209 & 4.12832299 & 3.37771881 \\
\hline 34 & Boxes crane cycle time & 0.3175065 & 0.349257238 & 0.285755922 \\
\hline 35 & Time after cargo handling operation & 950 & 1045 & 855 \\
\hline 36 & Number of cranes & 71 & 35 & 71 \\
\hline 37 & Hold foreman & 60 & 25 & 60 \\
\hline 38 & Quay foreman & 60 & 25 & 60 \\
\hline 39 & Hold workers & 373 & 130 & 373 \\
\hline 40 & Quay workers & 384 & 130 & 387 \\
\hline 41 & Bags hold worker & 57 & 25 & 57 \\
\hline 42 & Bags quay worker & 57 & 25 & 57 \\
\hline 43 & Crane man & 203 & 105 & 203 \\
\hline 44 & Hook man & 94 & 50 & 94 \\
\hline
\end{tabular}

Step 1: In this step, we divide the 44 factors into two subgroups. The first subgroup includes the first 20 factors; the factors related to the activities prior to the cargo handling process. The second subgroup contains the remaining 24 factors. Then, $Y(20)$ is calculated by setting the factors from 1 to 20 (the factors of the first subgroup) at their high level and the remaining factors (the factors of the second subgroup) at their low level. 
Table II: Observations for the first two extreme scenarios.

\begin{tabular}{|c|r|r|r|}
\hline Replicate $\boldsymbol{r}$ & $\mathbf{Y ( 0 )} \boldsymbol{r}$ & $\boldsymbol{Y ( 4 4 ) ;} \boldsymbol{r}$ & \multicolumn{1}{c|}{$\hat{\beta}_{\mathbf{1 - 4 4}}$} \\
\hline 1 & 6517.427 & 13198.371 & 3340.472 \\
\hline 2 & 4599.241 & 8253.074 & 1826.917 \\
\hline 3 & 6752.474 & 7466.298 & 356.912 \\
\hline 4 & 6147.086 & 10557.723 & 2205.319 \\
\hline 5 & 5905.501 & 10058.234 & 2076.367 \\
\hline 6 & 5436.791 & 6307.268 & 435.239 \\
\hline 7 & 5560.828 & 12640.537 & 3539.855 \\
\hline 8 & 6465.812 & 7096.785 & 315.487 \\
\hline 9 & 6536.732 & 9374.625 & 1418.947 \\
\hline 10 & 5844.715 & 8322.789 & 1239.037 \\
\hline Average & $\mathbf{5 9 7 6 . 6 6 1}$ & $\mathbf{9 3 2 7 . 5 7 0}$ & $\mathbf{1 6 7 5 . 4 5 5}$ \\
\hline Standard error & $\mathbf{2 0 7 . 1 0 8}$ & $\mathbf{7 2 8 . 2 8 0}$ & $\mathbf{3 6 6 . 5 9 3}$ \\
\hline $\boldsymbol{t}$-statistic & $\mathbf{2 8 . 8 5 8}$ & $\mathbf{1 2 . 8 0 8}$ & $\mathbf{4 . 5 7 0}$ \\
\hline Critical value $(\boldsymbol{\alpha}=\mathbf{0 . 1})$ & $\mathbf{1 . 3 8 3}$ & $\mathbf{1 . 3 8 3}$ & $\mathbf{1 . 3 8 3}$ \\
\hline
\end{tabular}

Simulation of 10 replicates gives the average turnaround time $\bar{Y}(20)=6648.184$ minutes. The aggregate main effects of the two subgroups are calculated as follows:

$$
\begin{aligned}
& \overline{\beta_{1-20}^{\wedge}}=\frac{\bar{Y}(20)-\bar{Y}(0)}{2}=335.762 \\
& \overline{\beta_{21-44}^{\wedge}}=\frac{\bar{Y}(44)-\bar{Y}(20)}{2}=1339.693
\end{aligned}
$$

Note that this step splits the total main effect $\overline{\hat{\beta}}_{1-44}=1675.455$ of the initial step into two additive components $\overline{\hat{\beta}}_{1-20}+\overline{\hat{\beta}}_{21-44}=335.762+1339.639=1675.455$. The $t$-statistic for the aggregate main effect of the first subgroup $\hat{\beta}_{1-20}$ is 2.608 and for the second subgroup $\hat{\beta}_{21-44}$ is 3.985. Therefore, the aggregate main effects of the two subgroups are significantly different from zero.

We continue splitting groups by the same way until we reach the important factors. We do not split a group any further if its estimated aggregate main effect is negative. For example, the group containing factors 29 to 32 and the group containing the factors 33 and 34 have negative aggregate main effect; -90.982 and -62.578 , respectively. The significant test is performed and it will be taken as a secondary criterion for splitting the groups. Fig. 1 shows the remaining steps of the sequential bifurcation. In this figure, the factors that have significant aggregate or individual main effects take light gray background, and the factors that have negative aggregate or individual main effects take dark gray background. The factors with zero effect take white background. Note that factor 41 is not included in the important factors' list because it has zero effect.

After 32 observations of the simulated response, we obtain 18 out of 44 important factors. Three of them (shown in bold in the table) have significantly different from zero main effects. These variables are the bags tackle load, the bags quay workers, and the number of inner launches. Table III shows the most important 18 factors in order of importance associated with their main effect and $t$-statistic. 


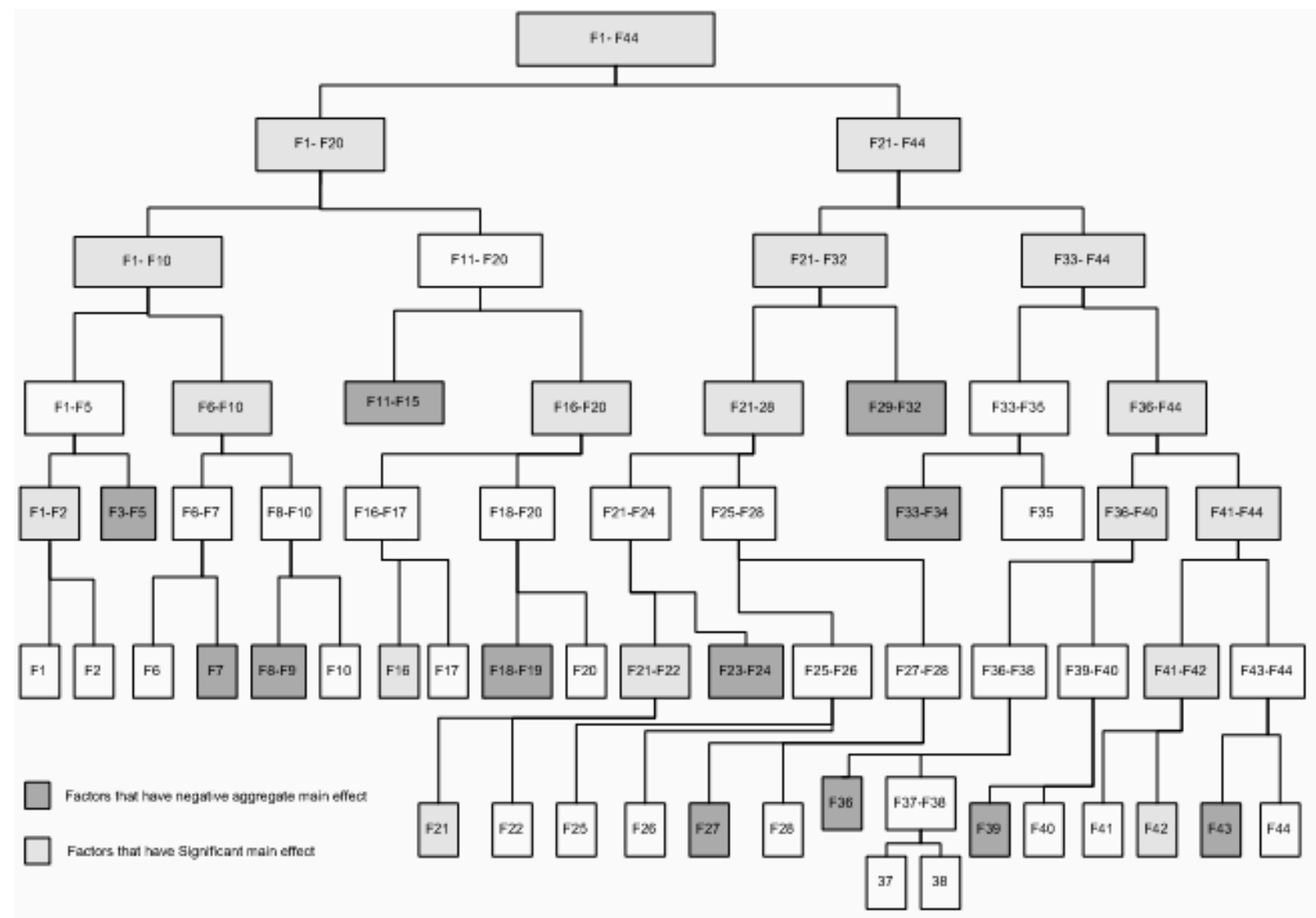

Figure 1: Sequential bifurcation steps with no interaction effect.

Cioppa [2] procedure can be used to verify that the assumed unimportant factors by the sequential bifurcation method are really unimportant. This procedure is used to confirm that the suspected non-influential (unimportant) factors do not significantly affect the interested response output. This procedure consists of two stages as follows:

Stage 1: Two simulation runs are performed. In the first run, all factors (important and unimportant) are set at their high level. In the second run, all factors are set at their low level. Let the turnaround time of the first run and the second run be $W_{1}$ and $W_{2}$ respectively; that is:

$$
\begin{aligned}
& W_{1}=\bar{Y}(44)=9327.57 \\
& W_{2}=\bar{Y}(0)=5976.661 .
\end{aligned}
$$

Let the difference between these two extreme values be $\delta_{1}=W_{1}-W_{2}=3350.909$.

Stage 2: It consists of two runs whose purpose is to verify that those factors determined as unimportant. In the first run, all important factors (18 factors) are set at their high level and all unimportant factors (26 factors) are set at their low level. Let $W_{3}$ be the turnaround from this run. In the second run set all important factors at their low level and all unimportant factors at their high level. Let $W_{4}$ be the turnaround time from this run. From these two scenarios, we obtain $W_{3}=8905.329$ and $W_{4}=6176.556$; then we calculate the following differences:

$$
\delta_{2}=W_{1}-W_{3}=422.241 \text { and } \delta_{3}=W_{4}-W_{2}=199.895
$$

If the set of unimportant factors really contains non-influential factors, then $\delta_{2}$ and $\delta_{3}$ should be small. In this case, we have four scenarios:

Scenario 1: if $\delta_{1}$ is large and both $\delta_{2}$ and $\delta_{3}$ are small, then this suggests that the unimportant factors are really unimportant. 
Scenario 2: if $\delta_{1}, \delta_{2}$ and $\delta_{3}$ are small, then all factors (important and unimportant) have no impact on the response variable (turnaround time).

Scenario 3: if $\delta_{1}$ is large and one or both $\delta_{2}$ and $\delta_{3}$ are large, then the unimportant set of factors may contain an important factor(s) that was not found by the sequential bifurcation method. In this case, more simulation runs should be conducted.

Scenario 4: if $\delta_{1}$ is small and one or both $\delta_{2}$ and $\delta_{3}$ are large, then the sequential bifurcation method may have incorrectly classified the factors.

Table III: The most important factors that affect the turnaround time without interaction.

\begin{tabular}{|c|c|l|r|r|}
\hline Rank & $\begin{array}{c}\text { Factor } \\
\text { number }\end{array}$ & \multicolumn{1}{|c|}{ Factors } & $\begin{array}{c}\text { Main effect } \\
\text { (minutes) }\end{array}$ & t-statistic \\
\hline 1 & 44 & Hook man (the most important) & 375.245 & 0.986 \\
\hline $\mathbf{2}$ & $\mathbf{4 2}$ & Bags quay workers & $\mathbf{3 4 9 . 9 5 0}$ & $\mathbf{1 . 5 9 8}$ \\
\hline 3 & 37 & Hold foreman & 290.656 & 1.236 \\
\hline $\mathbf{4}$ & $\mathbf{2 1}$ & Bags tackle load & $\mathbf{2 6 6 . 7 5 4}$ & $\mathbf{1 . 8 0 4}$ \\
\hline 5 & 40 & Quay workers & 265.876 & 0.874 \\
\hline 6 & 38 & Quay foreman & 187.455 & 0.559 \\
\hline 7 & 28 & Boxes tackle load & 1.343 \\
\hline 8 & 22 & Cartons tackle load & 143.832 & 0.739 \\
\hline 9 & 10 & Number of lashing launches & 118.278 & 1.151 \\
\hline 10 & 25 & Irons bars tackle load & 115.555 & 0.477 \\
\hline 11 & 20 & Time before cargo handling operation & $\mathbf{1 1 0 . 2 6 0}$ & $\mathbf{1 . 5 2 5}$ \\
\hline $\mathbf{1 2}$ & $\mathbf{1 6}$ & Number of inner launches & 92.475 & 0.911 \\
\hline 13 & 6 & Third outer pilot & 76.123 & 0.710 \\
\hline 14 & 2 & Outer anchorage setup time & 73.431 & 0.866 \\
\hline 15 & 35 & Time after cargo handling operations & 44.322 & 0.336 \\
\hline 16 & 1 & Speed of ships outside port & 16.823 & 0.108 \\
\hline 17 & 17 & Tugboats & 10.235 & 0.094 \\
\hline 18 & 26 & Iron rolls tackle load & & \\
\hline
\end{tabular}

Since $\delta_{1}$ is large (3350.909) and both $\delta_{2}(422.241)$ and $\delta_{3}$ (199.895) are small we are in the first scenario. Therefore, the unimportant set of factors determined by the sequential bifurcation method is really unimportant.

Now the assumption of no interaction between factors will be dropped. In this case, we will assume that the two-factor interaction is effective; that is

$$
\beta_{i, j} \neq 0 \text { for all } 1 \leq i<j \leq n
$$

Let $Y_{-}(j) ; r$ denote the simulated output response with the factors 1 to $j$ set at their low levels in replication $r$ and the remaining factors are set at their high levels so that $Y_{-}(j)$ is the mirror observation of $Y(j)$. In this case, the aggregate main effect and the individual main effects are calculated as following:

$$
\begin{aligned}
& \hat{\beta}_{i-j ; r}=\frac{\left(Y(j) ; r-Y \_(j) ; r\right)-\left(Y(i-1) ; r-Y \_(i-1) ; r\right)}{4} \\
& \hat{\beta}_{j ; r}=\frac{\left(Y(j) ; r-Y \_(j) ; r\right)-\left(Y(j-1) ; r-Y \_(j-1) ; r\right)}{4}
\end{aligned}
$$

In addition to the previous two assumptions, we make the following third assumption: 
Assumption 3: if a factor has no important main effect, then this factor does not interact with any other factor; that is

$$
\beta_{i}=0 \Rightarrow \beta_{i, j}=0 \quad \text { for all } i \neq j
$$

As before, the steps of the sequential bifurcation will start with the two extreme scenarios. In the first scenario, all factors are set at their low level and in the second scenario all factors are set at their high level. Then we apply Eqs. (9) and (10) to find the value of $\overline{\hat{\beta}_{1-44}}$. Note that:

$$
\bar{Y} \_(44)=\bar{Y}(0) \text { and } \bar{Y} \_(0)=\bar{Y}(44)
$$

Therefore, $\overline{\hat{\beta}}_{1-44}$ will be as before 1675.455 minutes. The procedure will continue as before, see Fig. 2. We will use the same output response $\bar{Y}(j)$ of the first case and we will generate the mirror observation $\bar{Y}_{-}(j)$ by setting factors 1 to $j$ at their low level instead of high level. Finally, we obtained 16 important factors instead of 18; six of them have significantly different from zero main effects. They are the paper rolls crane cycle time, the quay foreman, the hook man, the time after cargo handling operation, the bags quay worker and bags hold worker. Table IV shows the 16 important factors; where the significant factors are shown in bold.

By comparing the factors in Table III and Table IV, we conclude the following:

1. The number of important factors decreased from 18 to 16.

2. The number of significant factors (that have $t$-statistic greater than the critical value 1.383) increased from 3 to 6 .

3. Ten of the factors are important in the two cases (without interaction and with interaction). They are factors number 1, 2, 21, 22, 28, 35, 38, 40, 42 and 44.

4. The rank of the important factors changed because of the two-factor interaction effect. For example, without interaction effect the quay foreman factor has rank 6 in importance, while with the interaction effect its rank becomes 2 .

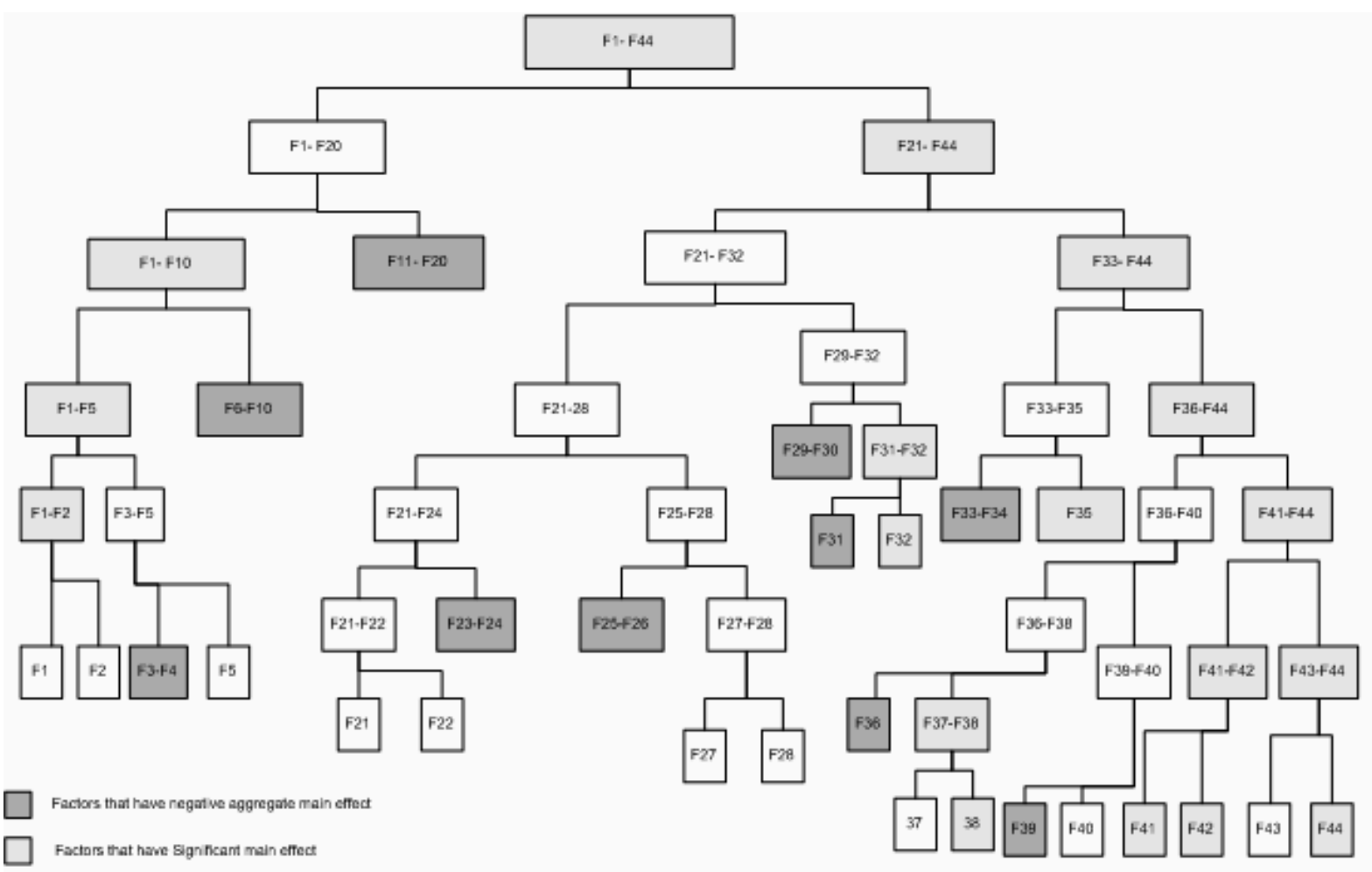

Figure 2: Sequential bifurcation steps with interaction effect. 
Table IV: Important factors that affect the turnaround time with two-factor interaction.

\begin{tabular}{|c|c|l|r|r|}
\hline Rank & $\begin{array}{c}\text { Factor } \\
\text { number }\end{array}$ & \multicolumn{1}{|c|}{ Factors } & $\begin{array}{r}\text { Main effect } \\
\text { (minutes) }\end{array}$ & t-statistic \\
\hline $\mathbf{1}$ & $\mathbf{3 2}$ & Paper rolls crane cycle time & $\mathbf{3 9 8 . 8 9 5}$ & $\mathbf{1 . 6 7 4}$ \\
\hline $\mathbf{2}$ & $\mathbf{3 8}$ & Quay foreman & $\mathbf{3 7 0 . 2 7 5}$ & $\mathbf{1 . 4 7 9}$ \\
\hline $\mathbf{3}$ & $\mathbf{4 4}$ & Hook man & $\mathbf{3 0 2 . 6 3 5}$ & $\mathbf{1 . 7 4 5}$ \\
\hline $\mathbf{4}$ & $\mathbf{3 5}$ & Time after cargo handling & $\mathbf{2 3 3 . 1 2 3}$ & $\mathbf{1 . 4 4 7}$ \\
\hline 5 & 2 & Outer anchorage set up time & 209.689 & 1.025 \\
\hline 6 & 43 & Crane man & 201.869 & 1.036 \\
\hline $\mathbf{7}$ & 37 & Hold foreman & 187.672 & 1.161 \\
\hline 8 & $\mathbf{4 2}$ & Bags quay worker & $\mathbf{1 7 4 . 9 7 7}$ & $\mathbf{1 . 5 9 8}$ \\
\hline 9 & 22 & Cartons tackle load & 171.132 & 0.911 \\
\hline 10 & 1 & Speed of ships outside the port & 160.961 & .697 \\
\hline 11 & 5 & Second outer pilot & 143.810 & 0.787 \\
\hline 12 & 40 & Quay workers & 132.938 & 0.874 \\
\hline $\mathbf{1 3}$ & $\mathbf{4 1}$ & Bags hold worker & $\mathbf{1 2 0 . 0 2 1}$ & $\mathbf{2 . 0 1 9}$ \\
\hline 14 & 27 & Iron pipes tackle load & 115.646 & 0.959 \\
\hline 15 & 28 & Boxes tackle load & 52.030 & 0.269 \\
\hline 16 & 21 & Bags tackle load & 31.267 & 0.163 \\
\hline
\end{tabular}

Since the individual main effect without two-factor interaction is different from the individual main effect with two-factor interaction, therefore, we conclude that the interaction between the factors is important.

\section{CONCLUSION}

The simulation model includes many controllable and uncontrollable (environmental) factors. According to the "parsimony principle", few factors are responsible for most of the effect on the response variable. Therefore, a screening policy is required to determine the most important factors. Many screening methods have been reviewed and the most appropriate one, the sequential bifurcation technique, is chosen to screen the simulation model. Forty-four controllable factors that affect the ship's turnaround time in a port are considered. The sequential bifurcation method is applied in two cases. In case one, we assumed no interaction effect between factors, a list of 18 important factors is obtained; three of them have statistically significant main effects. These factors are the bags quay workers; the bags tackle load, and the number of inner launches. In case two, two-way interaction effect is allowed. We obtained 16 important factors; six of them have statistically significant effect. Ten factors are common in the two cases.

The unimportant factors determined by the sequential bifurcation procedure are tested to verify if they are really unimportant or not. Cioppa [2] procedure is used to verify that the assumed unimportant factors by the sequential bifurcation method are really unimportant. This procedure confirmed that the set of unimportant factors really have no influence on the ship's turnaround time in the port.

Surprisingly, all the important factors are related to the cargo handling activities. These factors are the paper rolls crane cycle time, the quay foremen, the hook men, the time after cargo handling operations, the bags quay workers, and the bags hold workers. Therefore, the ship's turnaround time can be reduced by changing these factors in the appropriate direction instead of providing an additional berth or crane. This indicated that the operational efficiency of a seaport is not proportional to the number of berths in all cases. Consequently, for the port 
of Alexandria, in order to reduce the ship's turnaround time, there is no need to construct additional berths and spend millions of dollars. Instead, the port authority needs to hire the required cargo handling workers, reduce the crane cycle time by using qualified gangs and better cranes, or reduce the time that the ship stays at berth after finishing the cargo handling operations; for example, reduce the required time to finish the departure permission.

\section{ACKNOWLEDGEMENT}

The authors would like to thank the referees for carefully reading the first draft of the manuscript and for making numerous suggestions that improved the quality and readability of the paper.

\section{REFERENCES}

[1] Bettonvil, B.; Kleijnen, J. P. C. (1996). Searching for important factors in simulation models with many factors: Sequential bifurcation, European Journal of Operational Research, Vol. 96, 180194

[2] Cioppa, T. (2004). An efficient screening methodology for a priori assessed non-influential factors, Proceedings of Winter Simulation Conference, 711-715

[3] Trocine, L.; Malone, L. (2001). An overview of newer, advanced screening methods for the initial phase in experimental design, Proceedings of Winter Simulation Conference, 169-178

[4] Trocine, L.; Malone, L. (2000). Finding important independent variables through screening designs: A comparison of methods, Proceedings of Winter Simulation Conference, 749-754

[5] Kleijnen, J. P. C.; Bettonvil, B.; Person, F. (2005). Screening for the important factors in large discrete-event simulation models: Sequential bifurcation and its applications, Dean, A. M.; Lewis, S. M. (Editors), Screening, Springer-Verlag, New York

[6] Bettonvil, B. (1990). Detection of Important Factors by Sequential Bifurcation, Ph.D. dissertation, Tilburg University Press, Tilburg

[7] Kleijnen, J. P. C.; Sanchez, S.; Lucas, T.; Cioppa, T. (2005). A user's guide to brave new world of designing simulation experiments, Informs Journal on Computing, Vol. 17, No. 3, 263-289

[8] Cheng, R. C. H. (1997). Searching for important factors: Sequential bifurcation under uncertainty, Proceedings of the Winter Simulation Conference, 275-280

[9] Wan, H.; Ankenman, B.; Nelson, B. L. (2006). Controlled Sequential Bifurcation: A new factor screening method for discrete-event simulation, Operations Research, Vol. 54, No. 4, 743-75

[10] Sanchez, S. M.; Wan, H.; Lucas, T. (2005). A two-phase screening procedure for simulation experiments, Proceedings of Winter Simulation Conference

[11] Ragheb, M. A. (2005). A Decision Support Model for a Seaport, Ph. D. dissertation, Florida Institute of Technology

[12] Nelson, B. L. (1992). Designing efficient simulation experiments, Proceedings of Winter Simulation Conference, 126-132

[13] Donohue J. M. (1994). Experimental design for simulation, Proceedings of Winter Simulation Conference, 200-206

[14] Kleijnen, J. P. C. (1996). Five-stage procedure for the evaluation of simulation models through statistical techniques, Proceedings of Winter Simulation Conference, 248-254

[15] Kleijnen, J. P. C. (1997). Sensitivity analysis and related analysis: A review of some statistical techniques, Statistics and Computer Simulation, Vol. 57, 111-142

[16] Kelton, W. D. (2000). Experimental design for simulation, Proceedings of Winter Simulation Conference, 32-37

[17] Schmeiser, B. W. (2001). Some myths and common errors in simulation experiments, Proceedings of Winter Simulation Conference, 39-46

[18] Barton, R. R. (2002). Simulation experiments: designing simulation experiments, Proceedings of Winter Simulation Conference, 45-51

[19] Barton, R. R. (2004). Designing simulation experiments. Proceedings of Winter Simulation Conference, 73-79 